\title{
Equivalent linear systems of nonlinear systems
}

Friis, Tobias; Tarpø, Marius; Katsanos, Evangelos l.; Brincker, Rune

Published in:

Journal of Sound and Vibration

Link to article, DOI:

10.1016/j.jsv.2019.115126

Publication date:

2020

Document Version

Peer reviewed version

Link back to DTU Orbit

Citation (APA):

Friis, T., Tarpø, M., Katsanos, E. I., \& Brincker, R. (2020). Equivalent linear systems of nonlinear systems. Journal of Sound and Vibration, 469, [115126]. https://doi.org/10.1016/j.jsv.2019.115126

\section{General rights}

Copyright and moral rights for the publications made accessible in the public portal are retained by the authors and/or other copyright owners and it is a condition of accessing publications that users recognise and abide by the legal requirements associated with these rights.

- Users may download and print one copy of any publication from the public portal for the purpose of private study or research.

- You may not further distribute the material or use it for any profit-making activity or commercial gain

- You may freely distribute the URL identifying the publication in the public portal

If you believe that this document breaches copyright please contact us providing details, and we will remove access to the work immediately and investigate your claim 


\title{
Equivalent Linear Systems of Nonlinear Systems
}

\author{
Tobias Friis ${ }^{\mathrm{a}, *}$, Marius Tarp $\varnothing^{\mathrm{b}}$, Evangelos I. Katsanos ${ }^{\mathrm{a}}$, Rune Brincker $^{\mathrm{a}}$ \\ ${ }^{a}$ Technical University of Denmark, Department of Civil Engineering, Brovej B.118, Kgs. Lyngby, Denmark \\ ${ }^{b}$ Aarhus University, Department of Engineering, Inge Lehmanns Gade 10, Aarhus, Denmark
}

\begin{abstract}
The dynamic behaviour of civil engineering structures is frequently nonlinear, but it is often engineering practice to assume linearity. Consequently, a link between the nonlinear and linear models is required to ensure appropriate correspondence between the true dynamic behaviour and the model, which can be achieved by enforcing an equivalence between the two. This paper focuses on estimating equivalent linear systems of simulated multi-degree-of-freedom systems with stiffness and damping nonlinearities subjected to broad-band or general excitation using three different approaches based on different equivalences and assumptions. The considered methods are: a method based on balancing the intensity of the excitation and the response in the frequency domain, a modal version of the so-called energy dissipation method, and an approach that estimates equivalent linear stiffness, viscous damping, and equivalent linear mode shapes directly through a least squares fit. Notably, the latter method enables the assessment of the common assumptions such as low damping, slowly varying amplitude and phase, invariant mode shapes, and proportional damping. Furthermore, using the aforementioned procedures, extensive numerical simulations enable a comparative assessment in terms of the related linear stiffness, damping, and mode shape estimation potential of nonlinear systems and the accompanying error of the mentioned assumptions. In general, it is concluded that the methods provide a reliable estimation and interpretation of equivalent linear systems.
\end{abstract}

Keywords: Equivalent linear stiffness and viscous damping, equivalent linear mode shapes, equivalent linearisation, nonlinear dynamics, friction and hysteretic damping

\section{Introduction}

Nonlinear dynamics are present in engineering systems subjected to dynamic excitations. Different sources of nonlinearity can be identified and especially for structural systems in civil engineering, the phenomena of friction and hysteresis often occur [1,2]. Examples of such phenomena, frequently observed in civil engineering, could be friction between sliding surfaces, e.g. bolted joints, hysteresis in materials or geotechnical foundations, where the latter has decreasing stiffness with displacement [3]. Generally, however, the design and assessment of the dynamic performance of civil engineering structures are frequently based on linear assumptions and calculations that in many cases fall within an acceptable engineering precision. By introducing the rationale of equivalent linear systems, appropriate linear models are established based on nonlinear responses or nonlinear models, and subsequently, the established equivalent linear models are used as approximations of the corresponding nonlinear systems. Along these lines, research effort has been spent over the last $\sim 75$ years to elaborate on the equivalent linear stiffness and viscous damping for various nonlinear parametric models within the framework of both free and forced response, where the latter case mostly focusses on harmonic or random (Gaussian white noise) excitation.

The vast literature on the linear interpretation of nonlinear mechanical systems begins with the formulation of equivalent viscous damping of the steady-state response of single-degree-of-freedom (SDOF) systems

\footnotetext{
* Corresponding author

Email address: tofri@byg.dtu.dk (Tobias Friis)
} 
subjected to harmonic excitation introduced by Jacobsen [4]. The first formulation only covered velocity variation of the damping nonlinearity, however, Caughey [5] and Jennings [6] extended the method to cover both velocity and displacement variation. Besides harmonic excitation of SDOF systems, the immense topic of stochastic linearisation governs the literature with more than 400 papers and books, as Elishakoff [7] highligthed in his extensive literature review. The subject of stochastic linearisation is often divided into three categories: statistical linearisation, equivalent linearisation, and stochastic averaging. Booton [8] and Kazakov [9] originally proposed the statistical linearisation technique with subsequent extension to cover various aspects. For an overview of statistical linearisation, the reader is referred to [10]. The equivalent linearisation technique, which is oriented more towards mechanical systems, was introduced by Caughey [11-14] and consecutively generalised by Foster [15] and extended to multi-degree-of-freedom (MDOF) systems by Iwan and Yang $[16,17]$. Lastly, the stochastic averaging technique is generally contributed to Stratonovich [18], and resembles a linearisation method proposed by Kryloff and Bogoliubov [19]. The method has been described extensively and scrutinised by Roberts and Spanos [20] and Zhu [21]. Furthermore, the analytical procedure for the derivation of the linearised system parameters was significantly revised and refined in the late '90s by Elishakoff and Colajanni [22, 23] as well as by Bernard and $\mathrm{Wu}$ [24, 25]. For a review of the state-of-the-art in stochastic linearisation, the reader is referred to Socha and Soong [26] and Socha [27, 28]. Summarily, stochastic linearisation involves Gaussian white noise excitation and the assumption of slowly varying amplitude and phase, thus limiting the application to stochastic systems with low damping. Another approach was explored by Torres and Mote [29]. They proposed an iterative procedure based on averaging of the damping variation with the amplitude with respect to the probability density of the amplitudes, thus requiring not only iteration but also the assumption of slowly varying amplitude and phase. Additionally, the linear response characteristics of nonlinear systems have also been predicted by the application of perturbation techniques including, among others, the method of multiple scales consummated by Chuangdi et al. [30] and the harmonic balance method described by Worden and Tomlinson [31]. In this context, the objective is a prediction of the response level of a given system, where the present work is focussing on finding the equivalent linear system given a particular response of a nonlinear system. Lastly, an approach with a similar objective to the one of the present study can be found in the literature. With this approach a so-called best linear approximation is obtained by minimising $\mathrm{E}\left[|y(t)-g(t) * x(t)|^{2}\right], y(t)$ being the response, $g(t)$ being the impulse response function, $x(t)$ being the excitation, and $*$ the convolutional product [32-34]. The minimisation scheme is, however, only indirectly compliant with the desired definition of equivalence of the present paper.

Though the research effort within equivalent linear systems of nonlinear systems is vast, it is generally based on approximating assumptions such as harmonic or random excitation, low damping or constant amplitude and phase over one period of oscillation (i.e., slowly varying amplitude and phase), SDOF systems (i.e., invariant mode shapes for MDOF systems), and proportional damping. Therefore, the primary objective of the present paper is to provide methods for estimation and interpretation of equivalent linear systems of simulated nonlinear systems, spanning from simple calculations with several assumptions for less complex systems to more computationally heavy procedures without any assumptions for more complicated cases. The more computationally costly method enables the estimation of equivalent linear mode shapes. Moreover, the study also aims at illustrating the gross effect of employing the mentioned simplifying assumptions for cases where they are not entirely true.

Following this introduction, the paper presents the theoretical basis of three methods for estimation of equivalent linear systems, where the third method is employed in two different variations. Then the numerical simulation case is described, followed by extensive results from using the aforementioned methods. Eventually, the paper provides a comparative assessment in terms of the related equivalent linear stiffness, viscous damping, and equivalent linear mode shapes estimation potential with perspectives to their respective assumptions. It should be emphasised that the current study is based on friction and hysteretic systems in random vibrations, however, the presented methods are applicable for estimation of equivalent linear quantities of a vast variety of nonlinear systems in any kind of excitation. 


\section{Theoretical Basis of Methods for Estimation of Equivalent Linear Systems}

The methods employed herein are based on the definition of equivalent linearisation introduced by Caughey [11-14]. The concept of this approach is the formulation of a linear system that is equivalent to the nonlinear one in terms of forces. In mathematical form, the concept can be described in the following manner:

$$
\stackrel{\mathbf{M} \ddot{\mathbf{y}}_{n l}(t)+\mathbf{C} \dot{\mathbf{y}}_{n l}(t)+\mathbf{K} \mathbf{y}_{n l}(t)+\mathbf{f}_{n l}\left(\mathbf{y}_{n l}, \dot{\mathbf{y}}_{n l}\right)=\mathbf{x}(t)}{\stackrel{\text { interpretated as }}{\longrightarrow}} \quad \begin{aligned}
& \mathbf{M} \ddot{\mathbf{y}}_{n l}(t)+\mathbf{C}_{e q} \dot{\mathbf{y}}_{n l}(t)+\mathbf{K}_{e q} \mathbf{y}_{n l}(t)+\left.\mathbf{e}(t)\right|_{\text {min }}=\mathbf{x}(t)
\end{aligned}
$$

where $\mathbf{M}, \mathbf{C}$ and $\mathbf{K}$ are the mass, viscous damping and stiffness matrices, respectively, $\mathbf{f}_{n l}\left(\mathbf{y}_{n l}, \dot{\mathbf{y}}_{n l}\right)$ represents the nonlinear forces, $\mathbf{x}(t)$ is the external excitation, $\mathbf{y}(t)$ is the response, and $\mathbf{C}_{e q}$ and $\mathbf{K}_{e q}$ are the equivalent linear stiffness and viscous damping matrices. Furthermore, $\mathbf{e}(t)$ is a minimised error term describing the difference between the nonlinear system and the equivalent linear system, while $\left\{{ }^{\circ}\right\}$ denotes differentiation with respect to time $t$. The outcome of the aforementioned approaches has to be homogenised in order to allow their comparative assessment. Therefore, the damping is quantified by the use of the damping ratio, $\zeta$, which is a linear modal parameter widely adopted for various applications in the structural dynamics field, though, dependent on the equivalent stiffness.

\subsection{Method 1: Energy Balance}

Balancing the energy associated with the different terms of the equation of motion constitutes a widely used rationale to estimate the equivalent linear damping of dynamic systems. Along these lines, a modal version of the energy balancing method, initially introduced by Liang and Feeny [35-37], is proposed to estimate the total equivalent modal damping ratios for the case of general excitation. The proposed modal model resembles the traditional approach, where a nonlinear damping model is replaced by a linear viscous one with the same energy dissipation. Thus, it is referred to as the Energy Balance method. It is also notable that the Energy Balance method is associated with the least versatility compared to the two additional methods presented herein, since it can merely be employed for systems with nonlinear damping as the only nonlinearity.

To initiate a derivation of the first method introduced by the current study, the following equation is introduced describing the motion of an externally excited MDOF system with a general nonlinear damping term, $\mathbf{h}(\mathbf{y}, \dot{\mathbf{y}})$ :

$$
\mathbf{M} \ddot{\mathbf{y}}(t)+\mathbf{h}(\mathbf{y}, \dot{\mathbf{y}})+\mathbf{K y}(t)=\mathbf{x}(t)
$$

By multiplying all terms of Eq. (3) with $\dot{\mathbf{y}}^{T}(t)$ and integrating over a finite time interval, the following energy relation is obtained, in which the work of the dissipation forces, $W_{d i s}$, is equal to the difference between the work of the external, $W_{\text {ext }}$, and internal forces, $W_{i n t},[37]$.

$$
W_{d i s}=W_{e x t}-W_{i n t}
$$

The mathematical derivation of this method is facilitated by describing the general damping term of Eq. (3) as a viscous damping term, $\mathbf{h}(\mathbf{y}, \dot{\mathbf{y}}) \approx \mathbf{C}_{e q} \dot{\mathbf{y}}(t)$. This is similar to Eqs. (1) and (2), though, neglecting the error term:

$$
\mathbf{M} \ddot{\mathbf{y}}(t)+\mathbf{C}_{e q} \dot{\mathbf{y}}(t)+\mathbf{K y}(t)=\mathbf{x}(t)
$$

The current method and the following is formulated on the basis of modal components and the time-domain modal decomposition of the response, the latter being associated with the modal coordinates, $\mathbf{q}(t)$. When the mode shape matrix is well conditioned and the number of modes considered for the analysis is lower than the number of activated degrees-of-freedom (DOFs), the modal coordinates can be estimated as an overdetermined problem by using the Moore-Penrose (pseudo) inverse of the mode shape matrix [38]:

$$
\mathbf{y}(t)=\mathbf{B q}(t) \quad \Rightarrow \quad \hat{\mathbf{q}}(t)=\left(\mathbf{B}^{T} \mathbf{B}\right)^{-1} \mathbf{B}^{T} \mathbf{y}(t)
$$


where $\mathbf{B}$ is the mode shape matrix. Likewise, for the modal decomposition of the response, the excitation, $\mathbf{x}(t)$, can be transformed into the modal load: $\mathbf{p}(t)=\mathbf{B}^{T} \mathbf{x}(t)$. The equation of motion is further refined by its transformation into modal coordinates, provided by Eq. (6), while the multiplication of all the terms with the transposed mode shape matrix, $\mathbf{B}^{T}$, yields:

$$
\mathbf{B}^{T} \mathbf{M B} \ddot{\mathbf{q}}(t)+\mathbf{B}^{T} \mathbf{C}_{e q} \mathbf{B} \dot{\mathbf{q}}(t)+\mathbf{B}^{T} \mathbf{K B q}(t)=\mathbf{B}^{T} \mathbf{x}(t)
$$

Such a formulation of the equation of motion accommodates the diagonalisation of the inertia forces and restoring forces due to the orthogonality condition of the modes, and hence, they can be substituted by their modal components. Moreover, by assuming proportional damping, the viscous damping term also diagonalise [2, 39]:

$$
\mathbf{c}=\mathbf{B}^{T} \mathbf{C}_{e q} \mathbf{B}=2\left[\begin{array}{lll}
\zeta_{1} \omega_{1} m_{1} & & \\
& \ddots & \\
& & \zeta_{J} \omega_{J} m_{J}
\end{array}\right]
$$

where $m_{j}$ is the modal mass, $c_{j}$ is the modal dashpot coefficient, and $\omega_{j}$ is the natural angular frequency. The diagonalisation of all terms enables the formulation of the estimation equations from which the damping ratios can be readily estimated.

$$
\mathbf{m} \ddot{\mathbf{q}}(t)+2\left[\begin{array}{ccc}
\omega_{1} m_{1} \dot{q}_{1}(t) & & \\
& \ddots & \\
& & \omega_{J} m_{J} \dot{q}_{J}(t)
\end{array}\right]\left\{\begin{array}{c}
\zeta_{1} \\
\vdots \\
\zeta_{J}
\end{array}\right\}+\mathbf{k q}(t)=\mathbf{p}(t)
$$

where $\mathbf{m}$ and $\mathbf{k}$ are diagonal matrices of the modal mass, $m_{j}$, and modal stiffness, $k_{j}$. The transition to the energy-related equations is made by considering the individual, uncoupled modal equations of the aforementioned system (Eq. (9)), multiplying with $\dot{q}_{j}(t)$, and integrating over a finite time interval, $\tau=$ $t_{2}-t_{1}$ :

$$
m_{j} \int_{t_{1}}^{t_{2}} \dot{q}_{j}(t) \ddot{q}_{j}(t) \mathrm{d} t+2 \omega_{j} m_{j} \zeta_{j} \int_{t_{1}}^{t_{2}} \dot{q}_{j}^{2}(t) \mathrm{d} t+k_{j} \int_{t_{1}}^{t_{2}} \dot{q}_{j}(t) q_{j}(t) \mathrm{d} t=\int_{t_{1}}^{t_{2}} \dot{q}_{j}(t) p_{j}(t) \mathrm{d} t
$$

Finally, the system can be formulated on the basis of the energy balance initially expressed by Eq. (4), accounting for the unknown $\zeta_{j}$.

$$
\alpha_{j} \zeta_{j}=\beta_{j} \quad \Rightarrow \quad \zeta_{j}=\frac{\beta_{j}}{\alpha_{j}}
$$

where

$$
\begin{aligned}
& \alpha_{j}=2 \omega_{j} \int_{t_{1}}^{t_{2}} \dot{q}_{j}^{2}(t) \mathrm{d} t \\
& \beta_{j}=\int_{t_{1}}^{t_{2}} \dot{q}_{j}(t)\left(\frac{p_{j}(t)}{m_{j}}-\ddot{q}_{j}(t)-\omega_{j}^{2} q_{j}(t)\right) \mathrm{d} t
\end{aligned}
$$

Similarly to Eq. (4), the term $\alpha_{j} \zeta_{j}$ represents the work of the dissipation forces and $\beta_{j}$ represents the difference between the work of the external and internal forces.

\subsection{Method 2: Intensity Balance}

The second method presented herein is based on the relation between the intensity of the excitation and the response in the frequency domain. Hence, it will be referred to as the Intensity Balance method in the following. This method establishes relations between the response intensities, the magnitude of the random excitation, and the equivalent system parameters, and Caughey [14] exploited it to approximate the intensity of the displacement and velocity of the nonlinear system. In the present case, however, an alternative exploitation of the excitation-response intensity relation is pursued to allow for the estimation of equivalent linear stiffness and viscous damping quantities based on known response quantities. 
As described in the previous section, the modal decomposition of both the excitation and response enables the analytical formulation of the current method that assumes, similarly to the energy balance method, the mode shapes to be known and invariant. Moreover, the Intensity Balance method is based on the integration of the response spectral density, $S_{q_{j}}(\omega)$, of mode $j$ and the related expected value of the squared modal response, $\mathrm{E}\left[q_{j}^{2}\right]$, identical to the variance, $\sigma_{q_{j}}^{2}$, of the response assuming a zero mean process [39-41], i.e., Parseval's theorem.

$$
\mathrm{E}\left[q_{j}^{2}\right]=\sigma_{q_{j}}^{2}=\int_{-\infty}^{\infty} S_{q_{j}}(\omega) \mathrm{d} \omega
$$

where $\omega$ is the angular frequency. The use of the modal load spectral density, $S_{p_{j}}(\omega)$, allows us to rewrite the aforementioned formula as presented below.

$$
\sigma_{q_{j}}^{2}=\int_{-\infty}^{\infty}\left|H_{j}(\omega)\right|^{2} S_{p_{j}}(\omega) \mathrm{d} \omega
$$

where the frequency response function, $H_{j}(\omega)$, of the mode $j$ is provided by the equation below following a derivation on the basis of steady state responses of a system subjected by harmonic excitation.

$$
H_{j}(\omega)=\frac{1}{-m_{j} \omega^{2}+\mathrm{i} c_{j} \omega+k_{j}}
$$

Given the assumption that the modal load spectral density is broad-band and reasonably flat in the frequency range of the response band of the mode, $\omega_{e q(j)}$, an average value of $S_{p_{j}}(\omega)$ around $\omega_{e q(j)}$ can be used, and Eq. (14) reduces to an integration over a single function of $\omega$.

$$
\sigma_{q_{j}}^{2}=\int_{-\infty}^{\infty}\left|H_{j}(\omega)\right|^{2}\left\langle S_{p_{j}}\right\rangle_{\omega_{e q(j)}} \mathrm{d} \omega
$$

where \langle\rangle$_{\omega_{e q(j)}}$ denotes averaging around $\omega_{e q(j)}$.

The solution to Eq. (16) is readily provided by the relevant literature, e.g. [40, 42], and hence, the variance of the modal displacement response can be estimated by the following expression:

$$
\sigma_{q_{j}}^{2} \approx \pi \frac{\left\langle S_{p_{j}}\right\rangle_{\omega_{e q(j)}}}{2 \zeta_{e q(j)} \omega_{e q(j)}^{3} m_{j}^{2}}
$$

Like the derivation of the variance of the modal displacement response, the variance of the modal velocity response is obtained:

$$
\sigma_{\dot{q}_{j}}^{2} \approx \pi \frac{\left\langle S_{p_{j}}\right\rangle_{\omega_{e q(j)}}}{2 \zeta_{e q(j)} \omega_{e q(j)} m_{j}^{2}}
$$

Thereby, two equations have been obtained, Eqs. (17) and (18), for the determination of the two unknowns, $\zeta_{e q(j)}$ and $\omega_{e q(j)}$. Thus, the equivalent linear natural angular frequency (i.e., equivalent linear modal stiffness of mass normalised modal coordinates) and viscous damping are readily estimated by:

$$
\zeta_{e q(j)}=\pi \frac{\left\langle S_{p_{j}}\right\rangle_{\omega_{e q(j)}} \sigma_{q_{j}}}{2 \sigma_{\dot{q}_{j}}^{3} m_{j}^{2}}, \quad \omega_{e q(j)}=\frac{\sigma_{\dot{q}_{j}}}{\sigma_{q_{j}}}
$$

Based on the derivation, the method has one particular advantage in that the nonlinear response and the equivalent linear response, respectively, will share identical intensity (i.e., variance). Moreover, the natural frequency estimate of Eq. (19) is identical to the statistical average positive (or negative) zero crossing frequency of a narrow-banded process [40]. Such a process is characterised by a response resembling a sine wave with slowly varying amplitude and phase. When the damping is of a significant magnitude, the response is less distinct of such a sine wave and becomes more interrupted [41]. Thus, the estimates of the present method are expected to have a bias proportional to the magnitude of the damping since increasing damping changes the system from a narrow-banded to a broad-banded process. 


\subsection{Method 3: Direct Linear Fit in the Least Squares Sense}

The third method presented herein is based on a direct linear least squares fit of modal or physical parameters to the nonlinear response. This approach is similar to a procedure by Mohammad et al. [43], though further developed compared to the original. In the original context, the objective was to estimate the actual parameters of linear and nonlinear lumped parameter systems, whereas the purpose of the present efforts is to interpret and estimate linear quantities of nonlinear systems. Furthermore, both formulations in this paper allow for the extension from lumped parameter systems to discrete systems in configuration space as well as discrete and continuous systems in modal space. Lastly, the particular method agrees well with the definition of equivalent linearisation (Eqs. (1) and (2)). When employing this method, the error term of Eq. (2) is reduced by minimising the sum of the squares of the errors of the associated equations.

The first formulation of the method is based on modal components, which renders the implementation of the method rather straightforward and computationally efficient, and it is thus referred to as the Direct Linear Fit MS method in the following. However, the use of modal components leads to the assumption that the mode shapes are unaffected by the nonlinearity and that the damping is of the proportional type. Nonetheless, additional assumptions such as requirements of excitation type, linear inertia forces, and low damping are not necessary, whereby the method is associated with a higher versatility compared to the ones previously presented and to methods based on stochastic averaging, the latter assuming low damping. The second formulation, on the other hand, does not implement any assumptions but is more computationally heavy. It carries out a least squares fit of the full structural matrices and is thus referred to as the Direct Linear Fit CS method. By doing so, the method allows for the estimation of equivalent linear mode shapes by subsequently employing the eigenvalue problem on the estimated structural matrices.

\subsubsection{Method 3: Direct Linear Fit $M S$}

The first step of the third method formulated in modal space is related to considering the modal equation of motion of mode $j$ in Eq. (7):

$$
m_{j} \ddot{q}_{j}+c_{e q(j)} \dot{q}_{j}+k_{e q(j)} q_{j}=p_{j}
$$

The linear model, estimated from the nonlinear response, inherently adheres to the orthogonality condition with the modal decomposition in Eq. (6) and the diagonalisation of all structural matrices in Eq. (7) when assuming proportional damping. Thus, each mode can be considered individually. Furthermore, the modal equation of motion is rearranged as follows to allow the estimation of modal dashpot and stiffness coefficients of an equivalent linear system.

$$
p_{j}(t)-m_{j} \ddot{q}_{j}(t)=\left[\begin{array}{ll}
\dot{q}_{j}(t) & q_{j}(t)
\end{array}\right]\left\{\begin{array}{l}
c_{e q(j)} \\
k_{e q(j)}
\end{array}\right\}
$$

The main idea of the currently described method is to formulate Eq. (21) as an overdetermined linear least squares problem by utilising its discrete form in which every time step is considered as an equation. The least squares fit and its solution can formulated in the following manner [44].

$$
\mathbf{u}=\mathbf{X} \mathbf{a} \quad \Rightarrow \quad \hat{\mathbf{a}}=\left(\mathbf{X}^{T} \mathbf{X}\right)^{-1} \mathbf{X}^{T} \mathbf{u}
$$

where

$$
\mathbf{u}=\left\{\begin{array}{c}
p_{j}(1)-m_{j} \ddot{q}_{j}(1) \\
\vdots \\
p_{j}(N)-m_{j} \ddot{q}_{j}(N)
\end{array}\right\}_{N \times 1}, \quad \mathbf{X}=\left[\begin{array}{cc}
\dot{q}_{j}(1) & q_{j}(1) \\
\vdots & \vdots \\
\dot{q}_{j}(N) & q_{j}(N)
\end{array}\right]_{N \times 2}, \quad \mathbf{a}=\left\{\begin{array}{c}
c_{e q(j)} \\
k_{e q(j)}
\end{array}\right\}_{2 \times 1}
$$

and $N$ is the number of discrete points in the response series. If the modal mass is also an objective of the equivalent linear system estimation, the inertia forces can simply be included in the left hand side of Eq. (21) and successively in $\mathbf{X}$ and $\mathbf{a}$ as the damping and potential forces. Additionally, the residual of the least squares fit, provided by Eq. (24), can be used to assess the discrepancy of assuming linearity.

$$
\mathbf{r}=\mathbf{u}-\mathbf{X a ̂}
$$


The latter can be normalised by using the Coefficient of Determination:

$$
R^{2}=1-\sum_{n=1}^{N} r^{2}(n) / \sum_{n=1}^{N}(u(n)-\bar{u})^{2}
$$

where $\left\{^{-}\right\}$denotes the sample mean.

Fig. 1 shows an example of a linear fit of nonlinear damping forces for a mode with a linear damping ratio of $1.5 \%$ and an additional equivalent damping ratio of $\sim 11.75 \%$ stemming from Coulomb friction with stick-slip motion. The plot on the left depicts the principle of assuming linear viscous damping for a nonlinearly damped case, while the right plot shows the resulting modal damping forces over time. It is seen that the linearity assumption in this illustrative example case is crude, however, the principle and effect of the assumption are well depicted.
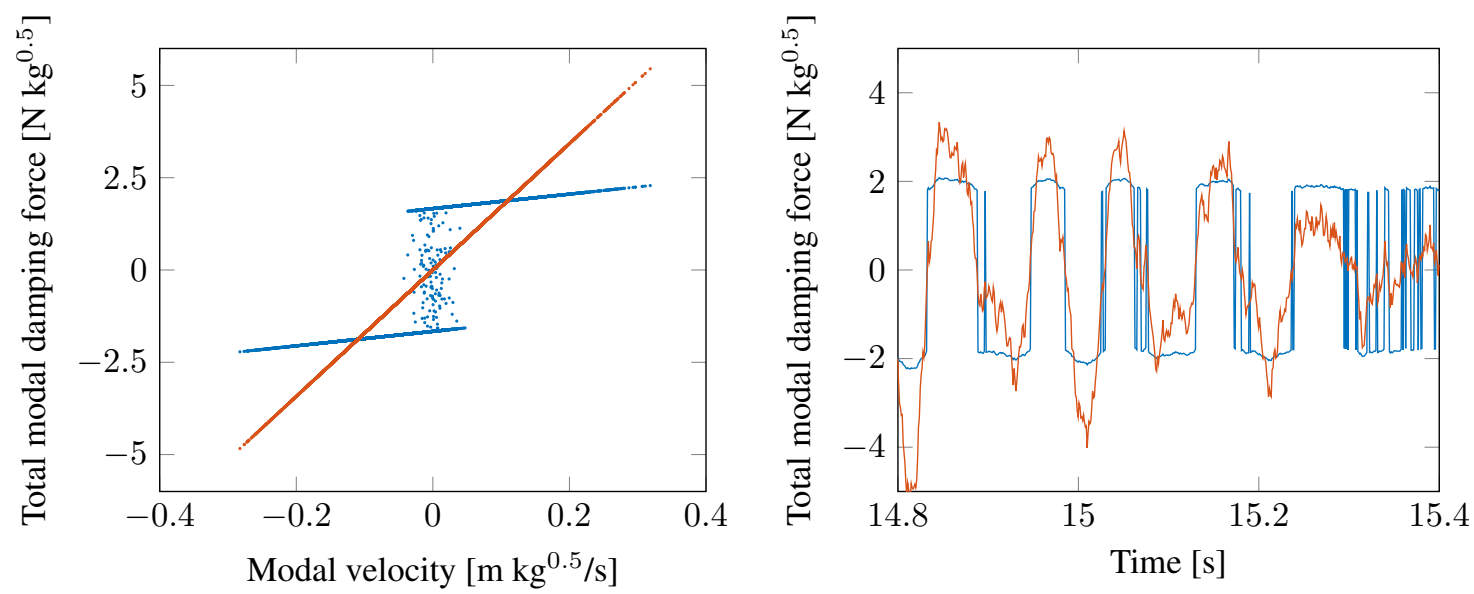

$$
\text { Actual nonlinear response quantity } \_ \text {Equivalent linear fit }
$$

Fig. 1 Example of a linear fit of modal damping forces from an example case of a mode with a linear damping ratio of $1.5 \%$ and an additional equivalent damping ratio of $\sim 11.75 \%$ stemming from Coulomb friction with stick-slip motion.

\subsubsection{Method 3: Direct Linear Fit CS}

As mentioned above, the second variation of the third method is based on a direct least squares linear fit and it is, contradictory to the first version (Section 2.3.1), formulated in configuration space. Practically, this implies a least squares linear fit of full structural matrices to the response, which yields a higher computational burden compared to the fitting of modal components to modal responses. By doing so, the last assumptions of invariant mode shapes with respect to the amplitude of the response and proportional damping are overcome.

Like Eq. (5), the equation of motion for a system with both equivalent linear stiffness and viscous damping can be described by the following equation.

$$
\mathbf{M} \ddot{\mathbf{y}}(t)+\mathbf{C}_{e q} \dot{\mathbf{y}}(t)+\mathbf{K}_{e q} \mathbf{y}(t)=\mathbf{x}(t)
$$

The subsequent derivation of the linear least squares fit is carried out for discrete systems with linear inertia forces and symmetric system matrices, indicated by SYM. The equation below shows the structure of the damping and stiffness matrices and their respective response vectors for a discrete system with $L$ degrees-of-freedom. 


$$
\mathbf{M} \ddot{\mathbf{y}}(t)+\left[\begin{array}{ccc}
c_{11} & \cdots & c_{1 L} \\
& \ddots & \vdots \\
\mathrm{SYM} & & c_{L L}
\end{array}\right]\left[\begin{array}{c}
\dot{y}_{1}(t) \\
\vdots \\
\dot{y}_{L}(t)
\end{array}\right]+\left[\begin{array}{ccc}
k_{11} & \cdots & k_{1 L} \\
& \ddots & \vdots \\
\mathrm{SYM} & & k_{L L}
\end{array}\right]\left[\begin{array}{c}
y_{1}(t) \\
\vdots \\
y_{L}(t)
\end{array}\right]=\mathbf{x}(t)
$$

Moreover, the least squares fit is formulated in a manner, where each position of the structural matrices is estimated. If more knowledge is known of the position of the individual contributions from the respective structural elements, the estimation equations can be readily refined to include equivalent linear quantities of the individual contributions. Similar to the formulation in modal coordinates, the structural terms of the equation of motion are rearranged to allow the estimation of physical dashpot and stiffness coefficients of an equivalent linear system.

$$
\begin{aligned}
& \mathbf{Y}_{r}(t)=
\end{aligned}
$$

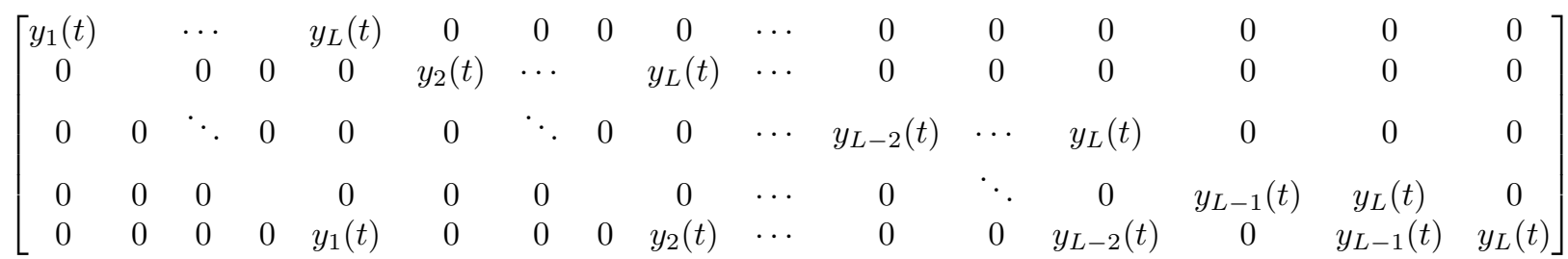

where $\mathbf{Y}_{r}(t) \in \mathbb{R}^{(L \cdot N) \times(L \cdot(L+1) / 2)}$ and

$$
\mathbf{c}_{c s}=\left\{\begin{array}{c}
c_{11} \\
\vdots \\
c_{1 L} \\
c_{22} \\
\vdots \\
c_{2 L} \\
\vdots \\
c_{L L}
\end{array}\right\}_{(L \cdot(L+1) / 2) \times 1} \quad, \quad \mathbf{k}_{c s}=\left\{\begin{array}{c}
k_{11} \\
\vdots \\
k_{1 L} \\
k_{22} \\
\vdots \\
k_{2 L} \\
\vdots \\
k_{L L}
\end{array}\right\}_{(L \cdot(L+1) / 2) \times 1}
$$

By rearranging the response components and the physical parameters, the equation of motion is provided by:

$$
\mathbf{x}(t)-\mathbf{M} \ddot{\mathbf{y}}(t)=\dot{\mathbf{Y}}_{r}(t) \mathbf{c}_{c s}+\mathbf{Y}_{r}(t) \mathbf{k}_{c s}
$$

As mentioned above, the inertia forces are assumed to be linear in this study and therefore not fitted to the response. In case they are nonlinear and equivalent forces are requested, the inertia forces are simply rearranged and the mass coefficients are estimated similarly to the damping and restoring forces.

Identical to the formulation in modal components, the discrete form is utilised with every time step, forming an equation. Thereby, the following least squares estimation of the physical parameters can be reached by the equations presented below.

$$
\mathbf{u}=\mathbf{X} \mathbf{a} \quad \Rightarrow \quad \hat{\mathbf{a}}=\left(\mathbf{X}^{T} \mathbf{X}\right)^{-1} \mathbf{X}^{T} \mathbf{u}
$$

where

$$
\mathbf{u}=\left\{\begin{array}{c}
\mathbf{x}(1)-\mathbf{M} \ddot{\mathbf{y}}(1) \\
\vdots \\
\mathbf{x}(N)-\mathbf{M} \ddot{\mathbf{y}}(N)
\end{array}\right\}_{(L \cdot N) \times 1} \quad, \quad \mathbf{X}=\left[\begin{array}{cc}
\dot{\mathbf{Y}}_{r}(1) & \mathbf{Y}_{r}(1) \\
\vdots & \vdots \\
\dot{\mathbf{Y}}_{r}(N) & \mathbf{Y}_{r}(N)
\end{array}\right]_{(L \cdot N) \times(L \cdot(L+1))} \quad, \quad \mathbf{a}=\left\{\begin{array}{l}
\mathbf{c}_{c s} \\
\mathbf{k}_{c s}
\end{array}\right\}_{(L \cdot(L+1)) \times 1}
$$


The discrepancy of assuming linearity can again be assessed through the residual in Eq. (24) and $R^{2}$ value in Eq. (25). With the definition of the equivalent linear system in Eqs. (1) and (2), the residual now equals the error term in Eq. (2), though in discrete form $\mathbf{e}(n)$.

When the physical parameters have been estimated, the system matrices in Eq. (27) are established. Subsequently, the natural frequencies, damping ratios, and mode shapes are readily found by solving the eigenvalue problem considering general damping. More details are provided by Brincker and Ventura [38].

\subsection{Summary of the Methods}

Three different methods for estimation of equivalent linear systems, where one was given in two alternative versions, have been presented and they all have different strengths and weaknesses. The Intensity Balance method has its strength in its simplicity, however, the stochastic nature leads, eventually, to a higher number of approximations compared to the other deterministic methods. The stochastic nature also introduces a link between the length of the response and the accuracy of the estimates, where the latter increases as the accuracy of the estimates of the response intensities and the spectral flat increases. Regarding the deterministic methods presented herein, i.e., the Energy Balance method and the Direct Linear Fit method, there is no dependency on the length of the response, and hence, these methods can be employed on responses with a duration shorter than a period of oscillation. Furthermore, the Energy Balance method is also readily implemented, efficient and based on an important physical aspect of a dynamic system, energy, but it is limited to the estimation of equivalent viscous damping. On the other hand, the main strength of the method based on a direct linear fit, is the versatility incorporated through the least squares fit, which enables the extension to estimation of equivalent linear mode shapes, general damping, and nonlinear inertia forces. Nonetheless, the wider the objectives of a method, the higher computational cost of the method and the more cumbersome is its employment. Categorically based on the background of the methods, neither of them are, from the authors perspective, more preferable compared to the others. The choice of method simply relies on the application. Table 1 summarises the required information and fundamental assumptions for the methods presented herein.

Table 1 Overview of the required information and fundamental assumptions for the three methods.

\begin{tabular}{|c|c|c|c|c|c|c|}
\hline Method & $\dot{\mathbf{y}}(t), \mathbf{y}(t)$ & $\ddot{\mathbf{y}}(t)$ & $\mathbf{x}(t)$ & B & $m_{j}$ & Assumptions \\
\hline Energy Balance & $\checkmark$ & $\checkmark$ & $\checkmark$ & $\checkmark$ & $\checkmark$ & $\begin{array}{l}\text { Invariant mode shape, pro- } \\
\text { portional damping }\end{array}$ \\
\hline Intensity Balance & $\checkmark\left(\sigma^{2}\right)^{*}$ & - & $\checkmark\left(\left\langle S_{p_{j}}\right\rangle_{\omega_{e q(j)}}\right)^{*}$ & $\checkmark$ & $\checkmark$ & $\begin{array}{l}\text { Invariant mode shape, low } \\
\text { and proportional damping, } \\
\text { broad-band excitation }\end{array}$ \\
\hline Direct Linear Fit MS & $\checkmark$ & $\checkmark$ & $\checkmark$ & $\checkmark$ & - & $\begin{array}{l}\text { Invariant mode shape, pro- } \\
\text { portional damping }\end{array}$ \\
\hline Direct Linear Fit CS & $\checkmark$ & $\checkmark$ & $\checkmark$ & - & - & - \\
\hline
\end{tabular}

* The exact information does not have to be known, but simply the information stated in the parentheses.

\section{Case Study: Numerical Models and Nonlinear Simulation}

\subsection{Numerical Model}

A numerical model of a T-shaped steel structure was created to provide the testbed for employing the aforementioned methods and evaluating them comparatively in terms of the equivalent linear quantities. First, a finite element model of the structural system without nonlinearities was developed through the employment of the commercial software ANSYS by using three dimensional beam elements with 12 degreesof-freedom (DOFs), each resulting in 156 DOFs in total. A common cross-section of SHS40x40x2 profile was adopted for the members of the structure, the latter modelled with fixed boundary conditions at the bottom 
(Fig. 2). No mid-side nodes were considered for the finite elements, and they were formulated by the use of the Timoshenko beam theory accounting for the shear deformation effects. The finite element model of the structural system considered here enabled the extraction of the mass, $\mathbf{M}$, and stiffness, K, matrices, which were reduced subsequently by the use of the System Equivalent Reduction and Expansion Process (SEREP) [45] to the 10 DOFs depicted in Fig. 2. The vibration frequencies of the first five vibration modes are listed in Table 2 along with the corresponding damping ratios of the linear system, assigned deliberately for simulation purposes. Finally, the numerical model was excited in all 10 DOFs by uncorrelated, Gaussian white noise, while the simulations were carried out with a time step of $0.00025 \mathrm{~s}$. The total simulation length is approximately equal to 15,000 periods of oscillation of the two lowest modes, i.e., $\sim 25$ min. The relatively large simulation length was chosen in order to reach similar statistical characteristics among the nonlinear response time histories.

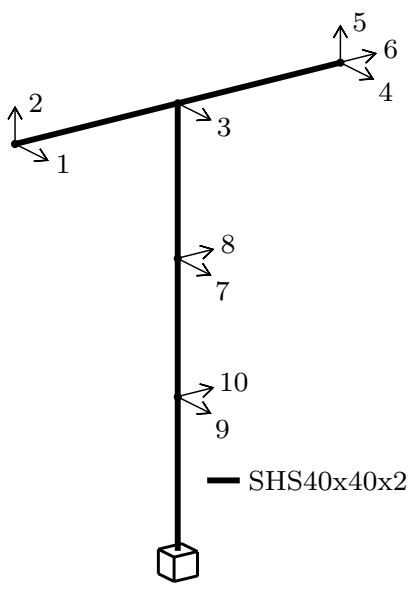

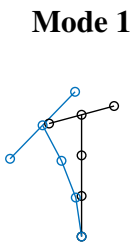

Mode 4

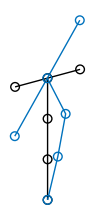

Mode 2

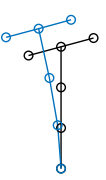

Mode 5

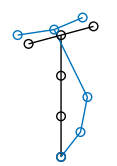

Fig. 2 Sketch of the simulated T-shaped structure with numbering of DOFs and mode shapes for the first five modes.

Table 2 Natural frequencies and damping ratios of the linear system.

\begin{tabular}{l|ccccc} 
Mode & 1 & 2 & 3 & 4 & 5 \\
\hline$f_{j}[\mathrm{~Hz}]$ & 10.10 & 10.39 & 27.58 & 57.54 & 93.27 \\
$\zeta_{\operatorname{lin}(j)}[\%]$ & 1.5 & 1 or 15 & 3.0 & 3.0 & 3.0
\end{tabular}

\subsection{Nonlinear Cases and Nonlinear Simulation}

In order to assess the methods for estimation of equivalent linear systems comparatively, three cases with different nonlinear behaviour were carefully chosen, see Fig. 3. In the first case, a Coulomb-type friction element was considered at the top of the T-shaped structure along DOF 3, affecting mainly the second of the first five modes. With this type of nonlinearity and its position, only the damping is varying with the amplitude, thus leaving the natural frequencies and the mode shapes practically constant. For the second analysis case, the nonlinearity was considered at the same location as the one chosen for the first analysis case. Hence, the nonlinearity mainly affects the second mode and it is modelled using the Iwan Model [46]. The latter is employed as four Jenkins elements in parallel and each one of them is composed of a spring in series with a Coulomb friction element of critical slipping force, i.e., a Jenkins element is an elasto-perfectly-plastic element. With the employment of this simple hysteresis model, the second case includes amplitude variation in both frequency and damping of the second mode, though, still leaving the mode shape practically invariant. Moreover, the third case is similar to the second one apart from applying an additional hysteresis-induced nonlinearity at the top of the T-shaped structure along DOF 4 . With this placement of the added nonlinearity, the mode shape of the second mode is no longer invariant with the amplitude of the response, thereby ensuring amplitude variation of all three modal quantities. Lastly, for 
the first case, a Coulomb friction force, $f_{c}$, of $4.0 \mathrm{~N}$ is considered. For the second and third case, the Iwan model is considered with Coulomb friction forces and spring constants of: $\mathbf{f}_{c}=[1.25,2.5,3.75,5] \mathrm{N}$ and $\mathbf{k}_{t}=[7,6,5,3.5] \cdot 10^{3} \mathrm{~N} / \mathrm{m}$. For all nonlinear cases, a linear damping ratio of $1 \%$ was considered for the second mode.

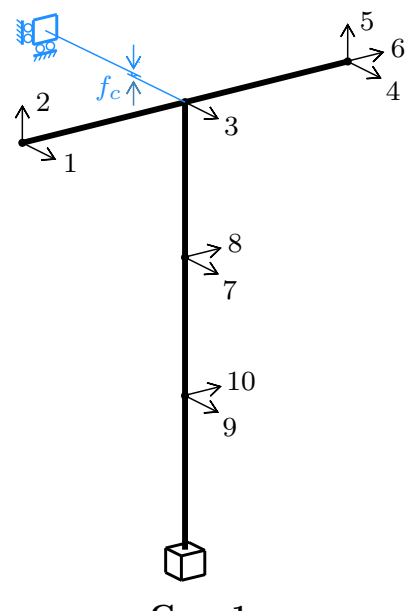

Case 1

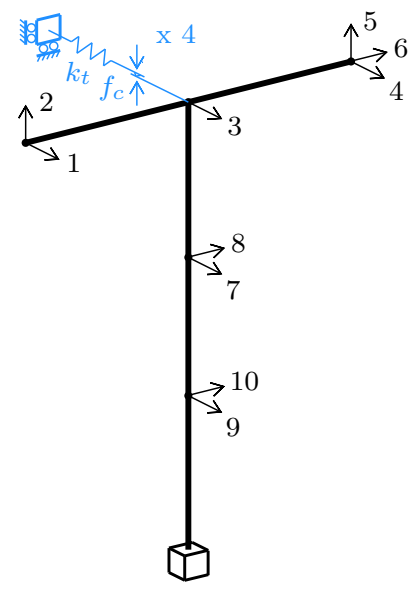

Case 2

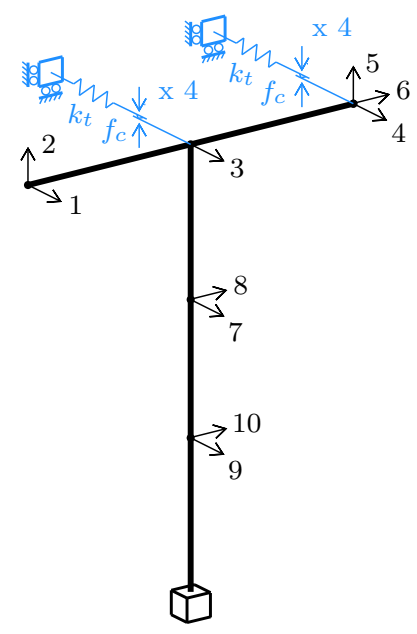

Case 3

Fig. 3 Depiction of the three nonlinear cases considered.

The simulation model dictates the formulation of the system of second-order differential equations for which the conventional linear system was preserved, including the terms that are associated with the inertia, viscous damping, stiffness, and external excitation. Additionally, the nonlinear forces were incorporated through an additional term, $\mathbf{D} \mathbf{f}_{d}(\mathbf{y}(t), \dot{\mathbf{y}}(t))$, and hence, the equation of motion is expressed as follows:

$$
\mathbf{M} \ddot{\mathbf{y}}(t)+\mathbf{C} \dot{\mathbf{y}}(t)+\mathbf{K y}(t)+\mathbf{D f}_{d}(\mathbf{y}(t), \dot{\mathbf{y}}(t))=\mathbf{x}(t)
$$

where $\mathbf{D}$ is the nonlinearity placement matrix and $\mathbf{f}_{d}(\mathbf{y}(t), \dot{\mathbf{y}}(t))$ is the nonlinear forces. The damping matrix is composed of the desired damping ratios of the modes considered herein, and the principles of proportional damping (Eq. 8.) were used to derive the terms of the $\mathbf{C}$ matrix [39]

$$
\mathbf{C}=\sum_{j=1}^{J}\left(\mathbf{M} \mathbf{b}_{j}\right) \frac{c_{j}}{m_{j}^{2}}\left(\mathbf{M b}_{j}\right)^{T}
$$

The dynamic analysis of the nonlinear system was carried out on the basis of the algorithm proposed by Lu et al. [47], and the related time stepping method was applied to redefine the equation of motion (Eq. (33)) in the following state space format:

$$
\dot{\mathbf{z}}(t)=\mathbf{A}_{c} \mathbf{z}(t)+\mathbf{D}_{c} \mathbf{f}_{d}(t)+\mathbf{E}_{c} \mathbf{x}(t)
$$

where $\mathbf{z}(t)$ represents the vector of state variables, $\mathbf{A}_{c}$ is the system matrix, while $\mathbf{D}_{c}$ and $\mathbf{E}_{c}$ are the counterparts of the nonlinearity placement matrix $\mathbf{D}$ and the excitation. The aforementioned components of the state space formulation are defined in the following:

$$
\begin{aligned}
\mathbf{z}(t)=\left[\begin{array}{l}
\dot{\mathbf{y}}(t) \\
\mathbf{y}(t)
\end{array}\right]_{2 L \times 1}, & \mathbf{A}_{c}=\left[\begin{array}{cc}
-\mathbf{M}^{-1} \mathbf{C} & -\mathbf{M}^{-1} \mathbf{K} \\
\mathbf{I} & \mathbf{0}
\end{array}\right]_{2 L \times 2 L} \\
\mathbf{D}_{c}=\left[\begin{array}{c}
\mathbf{M}^{-1} \mathbf{D} \\
\mathbf{0}
\end{array}\right]_{2 L \times V}, & \mathbf{E}_{c}=\left[\begin{array}{c}
\mathbf{M}^{-1} \\
\mathbf{0}
\end{array}\right]_{2 L \times L}
\end{aligned}
$$


where $\mathbf{I}$ is the identity matrix, $L$ denotes the number of DOFs, and $V$ denotes the number of dampers. It is notable that the solution to Eq. (35) is provided in discrete time format by assuming a linear variation of the nonlinear forces and the external forces between the successive time steps, i.e., first order hold. Furthermore, the nonlinear forces are dealt with by iterating the nonlinear forces from the attached elements within each time step. More details about the analytical solution adopted herein can be found elsewhere [47].

Fig. 4 illustrates the involved nonlinearities of the three cases considered. The figure includes approximately seven periods of the force-displacement relationship of the nonlinear elements.

Case 1

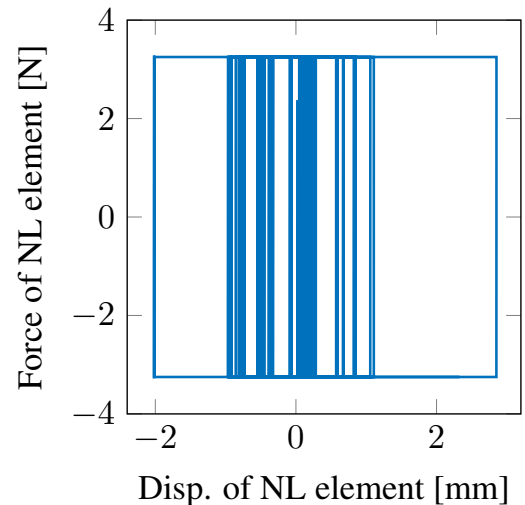

Case 2

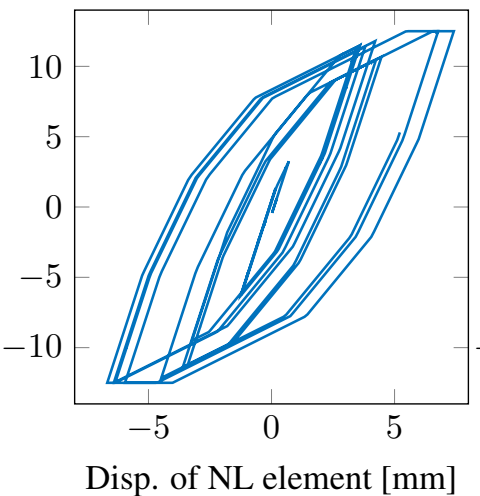

Case 3

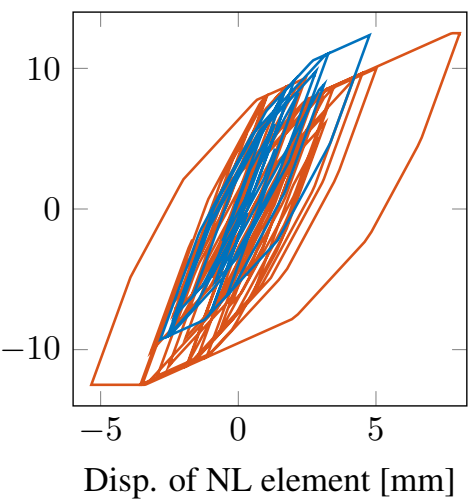

Fig. 4 Plot of approximately seven periods of the force-displacement relationship of the nonlinear elements in the three cases considered. (Case 3: blue; nonlinearity at DOF 3, red; nonlinearity at DOF 4)

\section{Case Study: Results}

The current section includes the demonstration and comparative assessment of the three methods presented herein considering the three cases, as described in Section 3.2, along with a linear baseline case. The linear baseline case is included in order to evaluate the performance of the methods without any presence of nonlinearities. The focus of the result section relates exclusively to the second mode of the numerical model since this mode is most severely affected by the nonlinearities in all cases. Furthermore, the estimates of linear stiffness and viscous damping were found to be Gaussian for all methods.

\subsection{Baseline: No Nonlinearities}

Regarding the linear baseline case, a damping ratio of $1 \%$ and $15 \%$ were considered for the second mode, respectively, stemming from the proportional viscous damping matrix. The choice of adopting two rather different damping ratios enables us to investigate the performance of the three proposed methods within the framework of the linear system that experiences both low and high damping. For both subcases, 500 Monte Carlo simulations were carried out by generating a new realisation of the random load for each simulation. The obtained estimation results of linear stiffness, shown in terms of natural frequency, and viscous damping, in terms of damping ratio, are plotted for the second mode in Fig. 5.

All methods were found to be able to estimate the linear input values reliably, though, the Intensity Balance method is associated with a statistical dispersion and low bias, where the latter is increasing with the damping magnitude. On the other hand, the dispersion of the frequency estimates is increasing with increasing damping, while the dispersion of the damping estimates is decreasing with increasing damping. More specifically, the coefficient of variation, i.e., the sample mean divided by the standard deviation, for the frequency estimates is equal to $0.0324 \%$ and $0.125 \%$ for the $1 \%$ and $15 \%$ damping, respectively. Contrary to this, the coefficient of variation of the damping estimates was found to be equal to $3.36 \%$ and $0.887 \%$ for the low and high damping case, respectively, thus delineating a relatively higher reliability in 
the frequency estimates than in the damping estimates. Lastly, the bias of the Intensity Balance method, which is increasing with the magnitude of the damping, is contributed to the method's inherent assumption of low damping in Eq. (19), as previously explained. Even in the case of a damping ratio of $15 \%$, the bias is found to be relatively low.
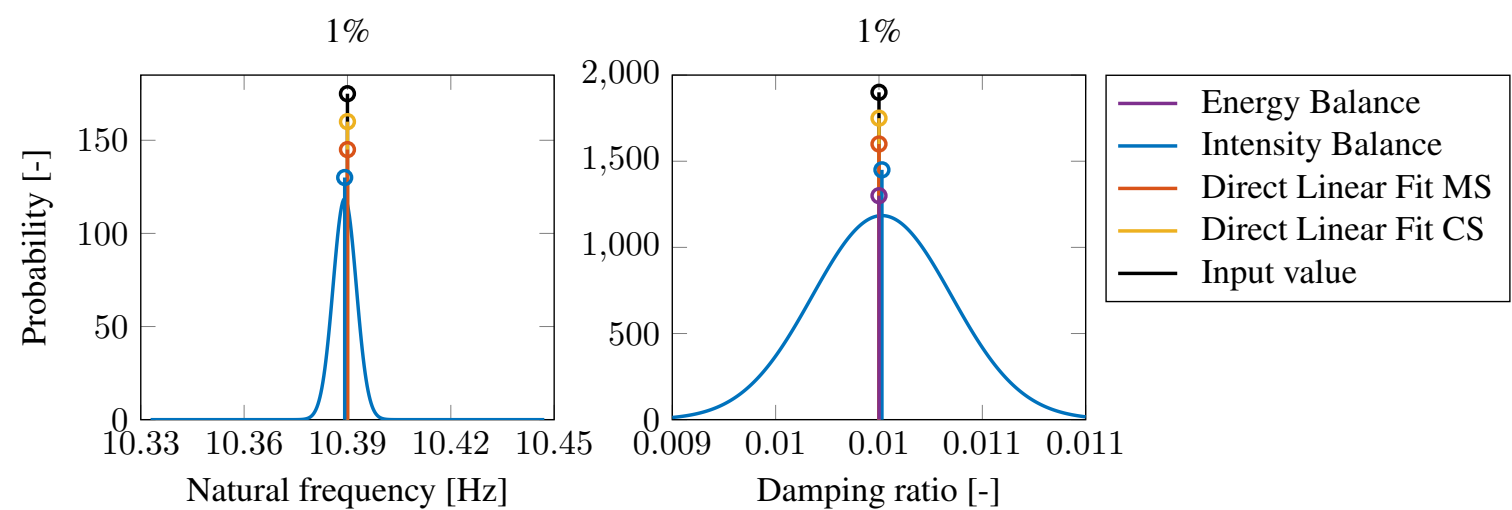

$15 \%$

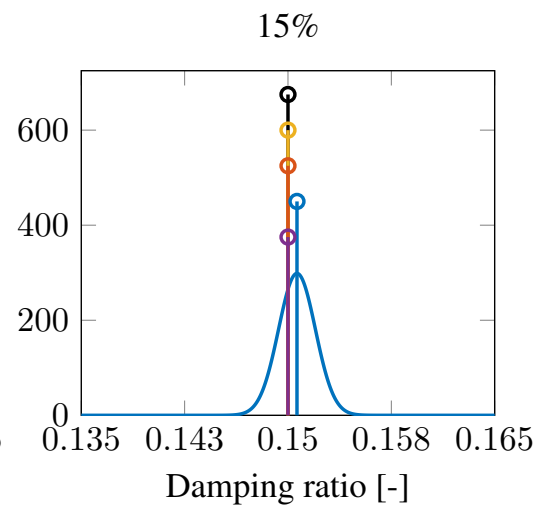

Fig. 5 Baseline: Estimation of linear frequency and viscous damping of the second mode from 500 linear simulations. Two subcases are considered: $1 \%$ and $15 \%$ damping of the second mode, top and bottom plot row, respectively.

\subsection{Case 1: Nonlinear Damping - Coulomb Friction}

As previously described, nonlinear damping in the form of Coulomb friction was considered for the first case. When dealing with equivalent linear stiffness and viscous damping of nonlinear systems, the equivalent system only describes the nonlinear system for the conditions for which it was established. Consequently, the three estimation methods were employed and their performance is demonstrated considering 18 response levels of the nonlinear system, and for each response level, 500 Monte Carlo simulations were carried out again by generating a new realisation of the random load for each simulation. The mean values of the obtained estimation results of natural frequency and damping ratio are plotted in Fig. 6 as a function of the mean modal response level of the second mode. The statistical dispersion is illustrated by a shaded area covering $\pm 2 \times$ the standard deviation $(\sigma)$. Furthermore, the figure is also depicting the kurtosis of the modal coordinates in terms of displacement as a function of the mean response level, illustrating the divergence of the distribution of the modal coordinates from the normal distribution, the latter being associated with a kurtosis value equal to 3.0. Additionally, the high quality of the fit, when employing the two variations of the Direct Linear Fit method is quantified and depicted as a function of the mean response intensity adopting the $R^{2}$-value (Eq. (25)). 
Comparing the estimation results presented in Fig. 6, all methods conclude with similar mean estimates of natural frequency and damping ratio, thus indicating reliability of the methods and the related estimated results. Nonetheless, when the damping is relatively high, a minor difference in the damping estimates can be observed. What might be difficult to see from Fig. 6 is that the estimates of all methods are characterised by a statistical dispersion with the largest dispersion of the Intensity Balance method. Even though the duration of the simulations was relatively long, the response will be different, ultimately culminating in slightly different equivalent quantities between Monte Carlo simulations. Analysing in detail the decimals of the estimates of the Energy Balance method and the Direct Linear Fit MS method, a perfect agreement is found. Based on this observation, it is concluded that estimating the equivalent viscous damping using the direct linear fit method is equivalent to establishing the equivalent viscous damping on the dissipated energy.
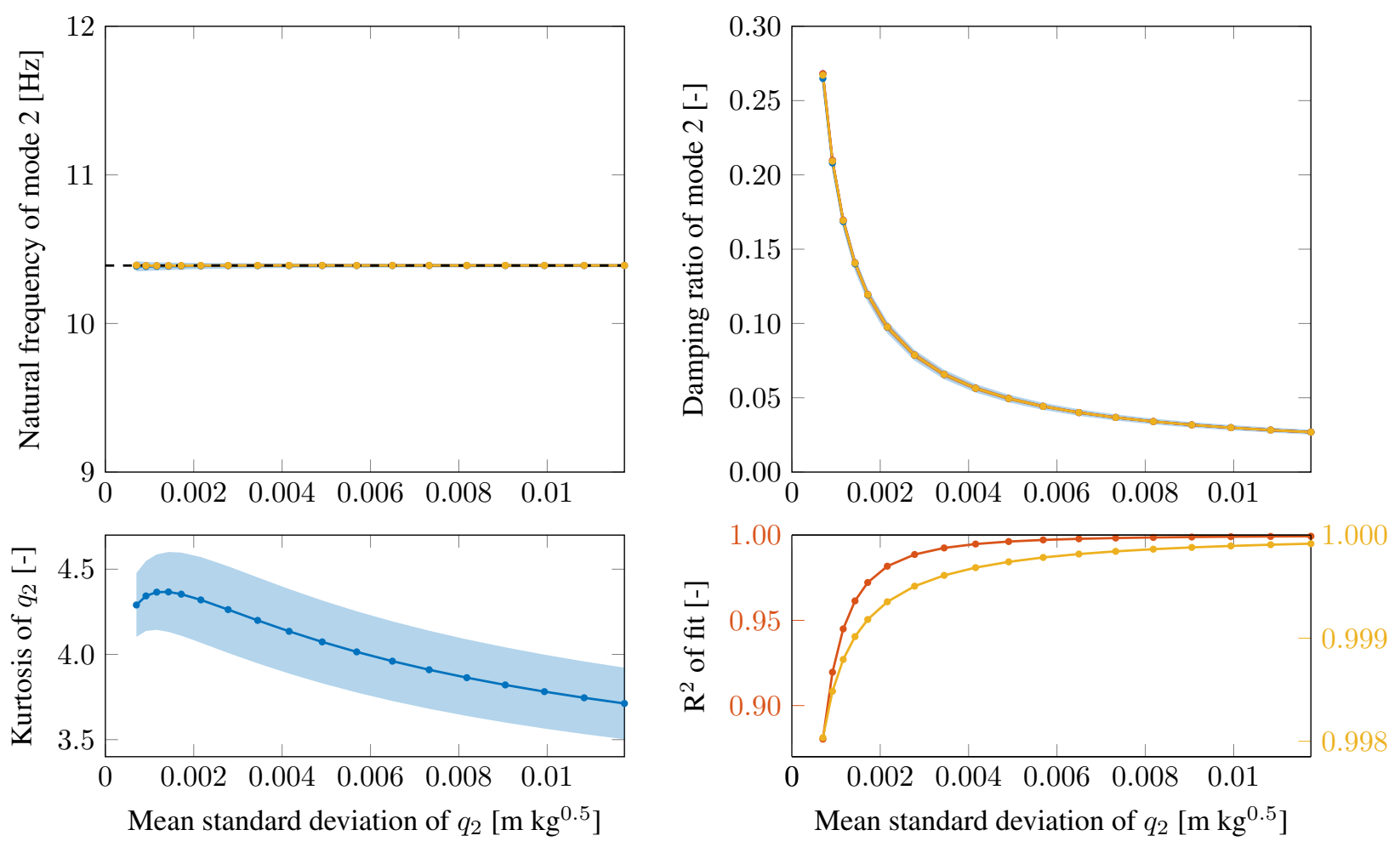

\begin{tabular}{lllll}
$\longrightarrow$ & Energy Balance & $\rightarrow$ & Intensity Balance & $\rightarrow$ Direct Linear Fit MS \\
\hdashline & Direct Linear Fit CS & $\cdots$ & Input natural freq. & \\
\hline
\end{tabular}

Fig. 6 Case 1: estimation results of the second mode for 18 response levels of 500 Monte Carlo simulations each. Shaded area is $\pm 2 \sigma$.

The relatively high kurtosis, i.e., larger than 3.0, is associated with a high probability density of the response at low amplitudes, and with more outliers at higher amplitudes deviating from the expected linear performance. Such a diversification is typical for a friction mechanism, where the low response amplitudes are associated with high damping forces and vice-versa. Moreover, Fig. 6 depicts the $R^{2}$-value calculated for the two variations of the Direct Linear Fit method as a function of the mean response intensity. The higher the damping is, the more nonlinear the damping becomes, thus the poorer the quality of the linear fit becomes. Based on this observation, the $R^{2}$-value can be used as a relative measure indicating how nonlinear the response is. Additionally, the $R^{2}$-values calculated for the Direct Linear Fit MS method are markedly lower compared to the $R^{2}$-values of the alternative variation in configuration space. This difference is not 
contributed to a fit of lower quality but to the difference in the formulation. The $R^{2}$-values of the Direct Linear Fit MS method consider only the nonlinearity affected second mode, where the $R^{2}$-values of the Direct Linear Fit CS method are accounting for all the vibration modes. Modes 1,3 and 4 are unaffected by the nonlinearity, which leads to $R^{2}$-values of the Direct Linear Fit CS method close to one.

The formulation of the Direct Linear Fit method in configuration space enables the estimation of an equivalent linear mode shape. The deviation or change in the mode shape obtained from the linear finite element model can be quantified using the Modal Assurance Criterion (MAC) [48]. In Fig. 7, the MACvalue of the second mode is plotted as a function of the corresponding mean response intensity, and the unity MAC-values demonstrate that the mode shape is not affected by the nonlinearity. Moreover, Fig. 7 reveals a mean error of the frequency and damping estimates as a function of the mean response intensity. This particular error is the difference between the estimates obtained by the two variations of the Direct Linear Fit method in modal space and in configuration space. Thus, the mean error is mainly related to the assumption of proportional damping and constant mode shapes. In the nonlinear case investigated in this section, the mode shape of the second mode is constant (Fig. 7), therefore, the relatively small error in the damping estimates at lower response levels can be related to the assumption of proportional damping.
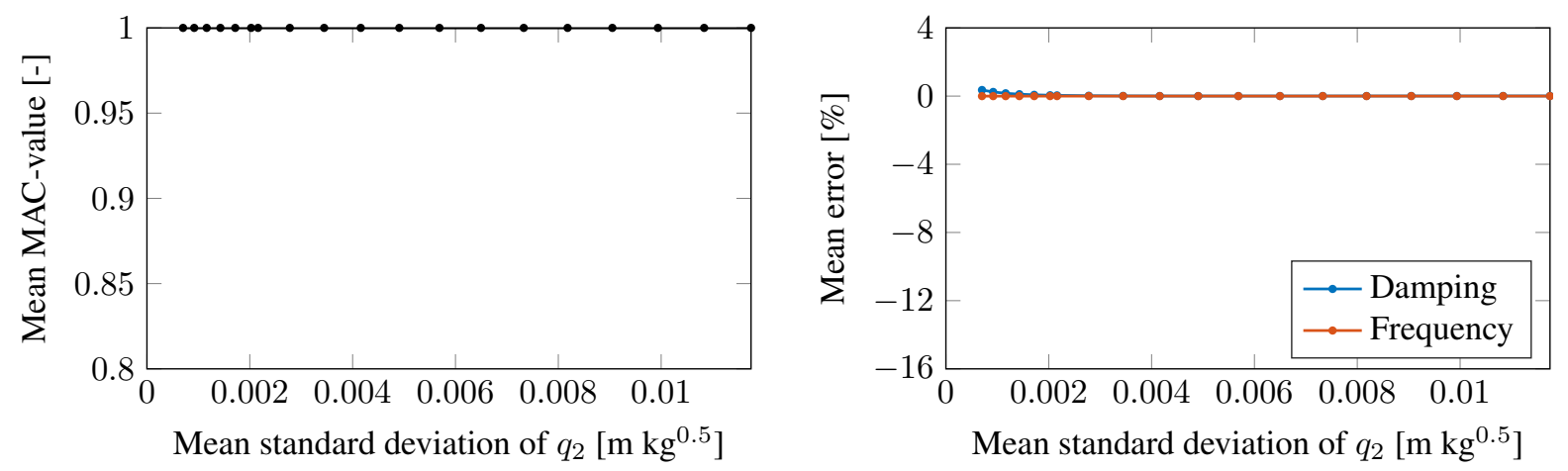

Fig. 7 Case 1: plot of MAC variation with the mean response intensity for the MAC between the second mode of the linear model and an equivalent linear mode shape of the second mode (left), and estimation errors relative to the assumptions of proportional damping and invariant mode shapes as a function of the mean response intensity (right).

\subsection{Case 2: Nonlinear Damping and Stiffness with Invariant Mode Shapes - Hysteresis}

Nonlinear damping and stiffness in the form of hysteresis were considered for the second case. The results of the second case are illustrated identically to the first case in Figs. 8 and 9. Contrary to the first case, for the second case, all of 29 response levels of the nonlinear system were considered, where 500 Monte Carlo simulations were carried out for each response level.

As for the first case, all methods conclude with similar estimates of the natural frequency and damping ratio, though with a larger statistical dispersion of the Intensity Balance method. Additionally, the natural frequency of the second mode also varies with the amplitude of the response. At a low response level, the natural frequency estimates progress asymptotically toward a situation with only elastic deformation of the nonlinear element, and at a high response level, the natural frequency estimates progress asymptotically toward a situation with no nonlinearity. The natural frequency of these two boundary states can be obtained by the eigenvalue problem of the linear model considering the initial stiffness of the nonlinearity and no nonlinearity, respectively (marked by dashed lines in Fig. 8). Moreover, the Energy Balance method excludes estimates of varying frequency, therefore, damping estimates have been carried out considering the natural frequency of these two boundary states, respectively. A considerable difference is discovered between the two obtained equivalent viscous damping curves for the Energy Balance method. 

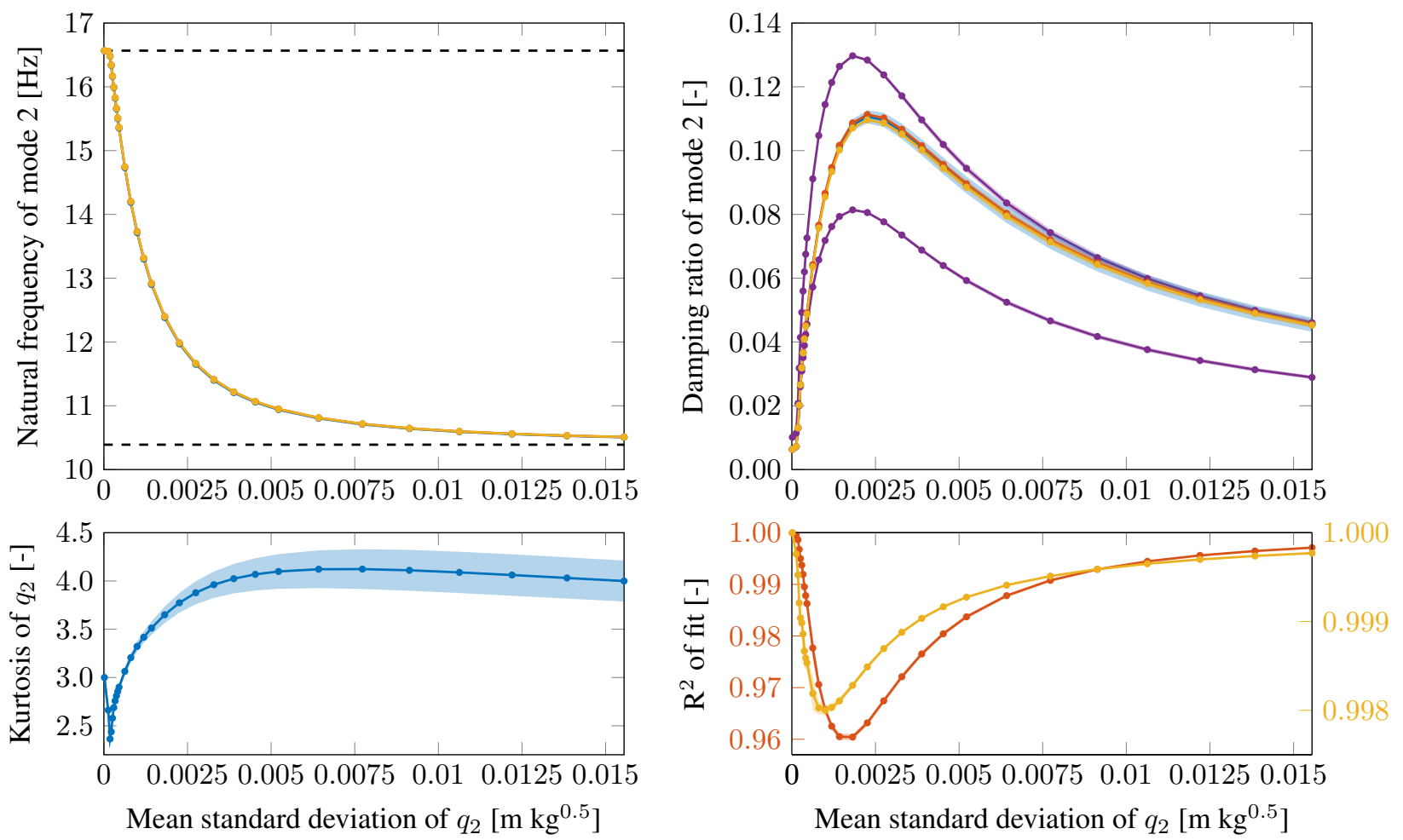

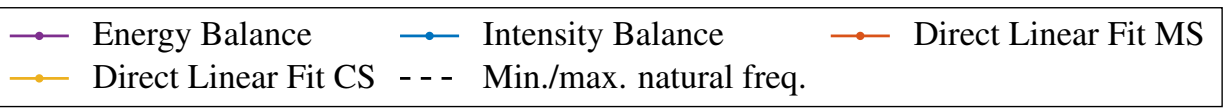

Fig. 8 Case 2: estimation results of the second mode for 29 response levels of 500 Monte Carlo simulations each. Shaded area is $\pm 2 \sigma$.
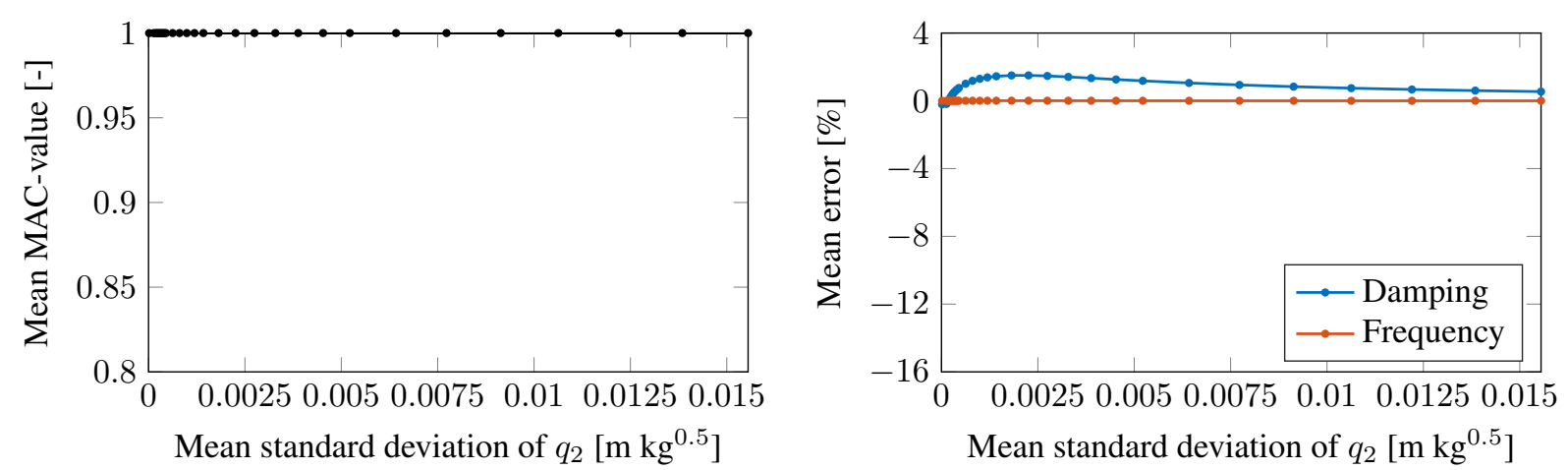

Fig. 9 Case 2: plot of MAC variation with the mean response intensity for the MAC between the second mode of the linear model and an equivalent linear mode shape of the second mode (left), and estimation errors relative to the assumptions of proportional damping and invariant mode shapes as a function of the mean response intensity (right).

The variation of the kurtosis of modal response of the second mode $\left(q_{2}(t)\right)$ with the mean response intensity also differs from the first case (Fig. 9). When the response intensity is relatively low, the kurtosis 
is less than 3.0, and for higher response intensities, the kurtosis is higher than 3.0. This particular behaviour suggests that the stiffness nonlinearity is dominating at lower response levels, whereas the damping nonlinearity is dominant for higher response levels. Furthermore, the variation of the $R^{2}$-value with the response intensity also diverges from the first case. As can be seen in Fig. 9, the $R^{2}$-value is one at a response intensity relatively close to zero, and progressing asymptotically towards one at higher response levels. This variation in the $R^{2}$-values illustrates that the nonlinear model is performing as a linear one when the response is within the elastic deformation of the nonlinear element, and as asymptotically linear when the dynamic forces of the linear part of the model are large compared to the forces of the nonlinear element.

\subsection{Case 3: Nonlinear Damping and Stiffness with Varying Mode Shapes - Hysteresis}

Response dependent damping, stiffness, and mode shapes were considered for the third case, as previously described, in the form of localised hysteresis placed at two locations of the T-shaped structural model. The estimation results of the third case are illustrated identically to the first and second case in Figs. 10 and 11.
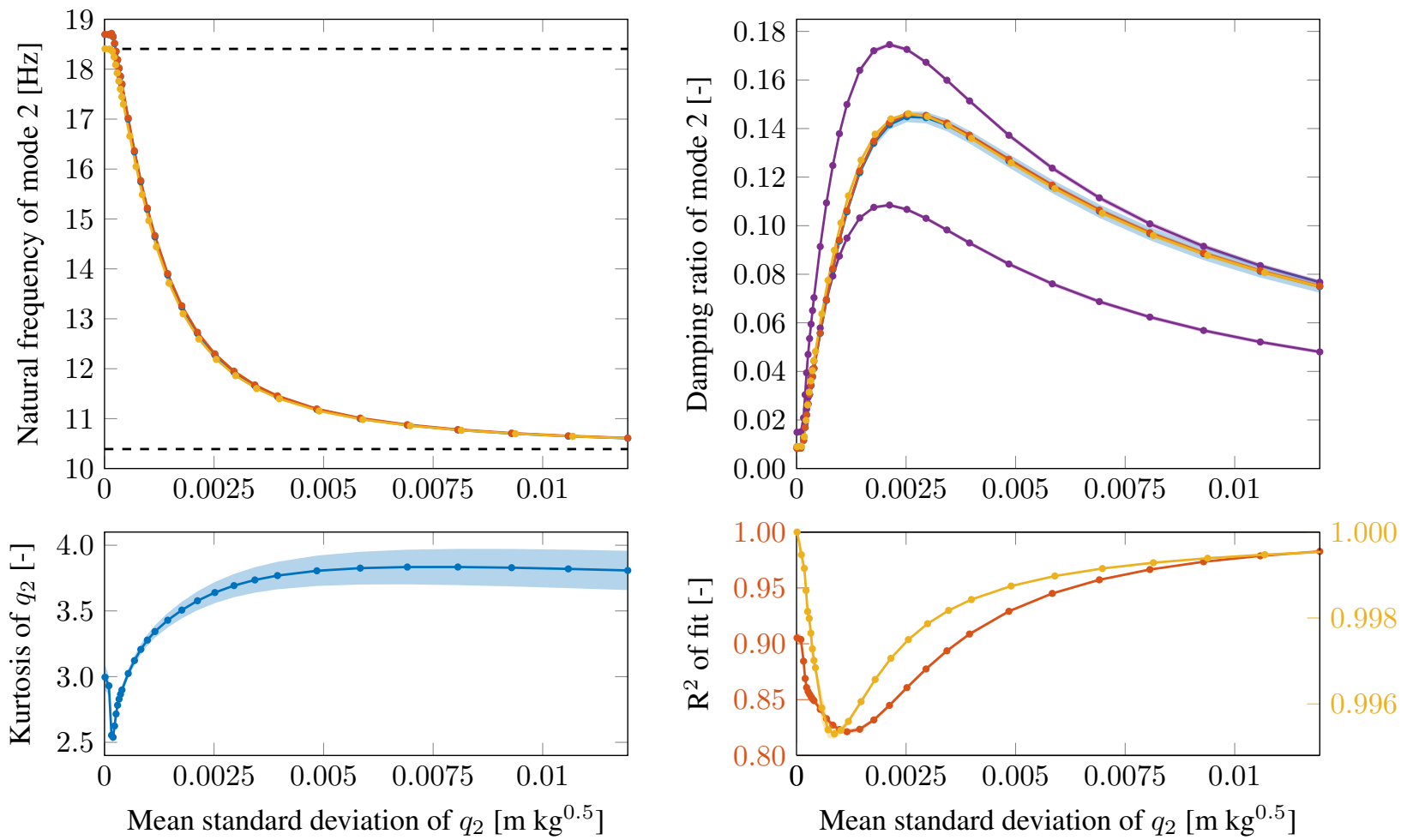

\begin{tabular}{llllll}
$\longrightarrow$ & Energy Balance & $\rightarrow$ & Intensity Balance & $\cdots$ & Direct Linear Fit MS \\
\hdashline & Direct Linear Fit CS & -- & Min./max. natural freq.
\end{tabular}

Fig. 10 Case 3: estimation results of the second mode for 29 response levels of 500 Monte Carlo simulations each. Shaded area is $\pm 2 \sigma$.

Comparing the main results of the third case in Fig. 10 to the main results of the second case in Fig. 8, the resemblance is uncanny. Additionally, the variation of the mean MAC-value and the estimation errors in Fig. 11 clearly reveal that the mode shape varies with the response amplitude for the present case. The error on the estimates of the natural frequency and damping ratio of the second mode peaks at $2.6 \%$ and $15.5 \%$, respectively, for the present case. Such an observation for the mean error is case dependent and 
hence cannot be readily generalised for other nonlinear systems. Notably, the errors on both the natural frequency and the damping increase with decreasing MAC-value, except for response levels close to zero. Based on these findings, error can be manifested in the estimation of equivalent linear systems when the estimation of equivalent mode shapes is excluded.
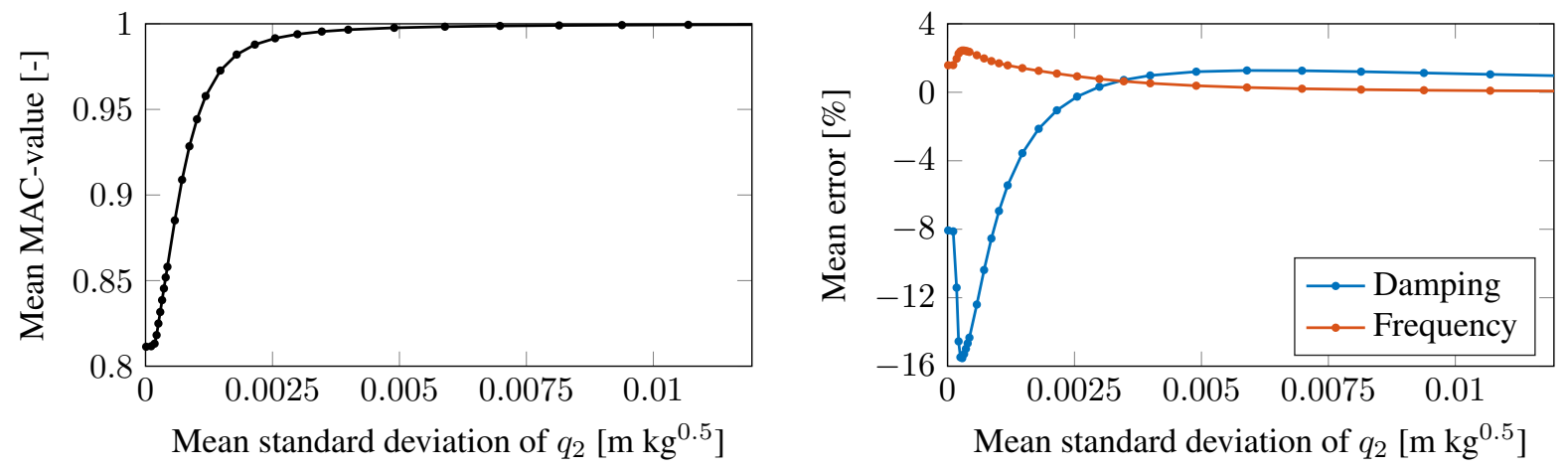

Fig. 11 Case 3: plot of MAC variation with the mean response intensity for the MAC between the second mode of the linear model and an equivalent linear mode shape of the second mode (left), and estimation errors relative to the assumptions of proportional damping and invariant mode shapes as a function of the mean response intensity (right).

It is important to mention that the particular estimates of the equivalent linear system are only truly valid for the conditions for which they were estimated. If the excitation is different, the same will apply to the equivalent linear system since the system is in fact nonlinear. No matter how the equivalence is formulated, the equivalent linear system will always be relative to the conditions for which it was estimated. Nonetheless, the performance of the proposed methods will naturally be similar to the performance in the demonstration cases above when applied to new cases. Even though the results obtained herein are less general, the application of the proposed methods is still general.

\section{Conclusion}

The objective of this study was to introduce robust methods for reliable estimation and interpretation of equivalent linear stiffness, viscous damping, and equivalent linear mode shapes of simulated nonlinear systems. For this purpose, three methods were presented that gradually decrease the number of employed assumptions, but increase the computational endeavour. Successively, the three methods were extensively tested through Monte Carlo simulations of a numerical model of a T-shaped structure. The methods were demonstrated and tested on the numerical model considered in one linear case (i.e., baseline) and in three different nonlinear cases, gradually increasing the number of nonlinear modal parameters. The simulation study enabled a comparative assessment of the equivalent linear system estimation potential and the inherited assumptions from which the following main conclusions are made:

- The methods presented herein were found to provide, in general, a reliable estimation and interpretation of equivalent linear systems in terms of equivalent linear stiffness, viscous damping, and equivalent linear mode shapes.

- The assumption of low damping in the derivation of the Intensity Balance method induced negative bias in the estimation of equivalent linear stiffness, and positive bias in the estimation of viscous damping. The introduced bias is increasing with increasing damping but it is relatively small.

- The inherited assumption of proportional damping implemented errors in the estimation of the equivalent linear quantities in conditions with high and localised damping. Again, the errors are relatively small. 
- For the third nonlinear case in which the mode shapes were varying with the intensity of the response, the assumption of invariant mode shapes led, as expected, to an erroneous estimation of the equivalent linear stiffness and the viscous damping, the latter being affected the most. The error can occur at a considerable magnitude.

\section{Acknowledgements}

The authors acknowledge the funding received from the Centre for Oil and Gas - DTU/Danish Hydrocarbon Research and Technology Centre (DHRTC).

\section{References}

[1] T. T. Soong, G. F. Dargush, Passive Energy Dissipation Systems in Structural Engineering, John Wiley \& Sons Ltd, 1997.

[2] A. K. Chopra, Dynamics of Structures, Pearson Education, 2012.

[3] M. Gunaratne, The Foundation Engineering Handbook, CRC Press, 2013.

[4] L. Jacobsen, Steady forced vibration as influenced by damping, American Society of Mechanical Engineers - Advance Papers (1930).

[5] T. K. Caughey, Sinusoidal excitation of a system with bilinear hysteresis, Journal of Applied Mechanics 27 (4) (1960) 640-643.

[6] P. Jennings, Equivalent viscous damping for yielding structures, American Society of Civil Engineers - Proceedings 94 (1968) 103-116.

[7] I. Elishakoff, P. Colajanni, Stochastic linearization critically re-examined, Chaos, Solitons \& Fractals 8 (12) (1997) 1957 -1972 .

[8] R. C. Booton, Nonlinear control systems with random inputs, Transactions of the Institute of Radio Engineers Professional Group on Circuit Theory CT-1 (1) (1954) 9-18.

[9] I. Kazakov, An approximate method for the statistical investigation for nonlinear systems, Trudj Voenno-Vozdushnoi Inzhenernoi Akademii imeni Prof. N.E. Zhukovskogo 4 (1954) 3-52.

[10] I. N. Sinitsin, Methods of statistical linearization (survey), Automation and Remote Control 35 (5) (1974) $765-776$.

[11] T. K. Caughey, Response of a nonlinear string to random loading, Journal of Applied Mechanics 26 (1959) $341-344$.

[12] T. K. Caughey, Response of van der pol's oscillator to random excitation, Journal of Applied Mechanics 26 (1959) 345-348.

[13] T. K. Caughey, Random excitation of a system with bilinear hysteresis, Journal of Applied Mechanics 27 (4) (1960) 649.

[14] T. K. Caughey, Equivalent linearization techniques, Journal of the Acoustical Society of America 35 (11) (1963) $1706-1711$.

[15] E. T. Foster, Semilinear random vibrations in discrete systems, Journal of Applied Mechanics 35 (1968) 560-564.

[16] W. D. Iwan, I.-M. Yang, Statistical linearization for nonlinear structures, Journal of the Engineering Mechanics Division 97 (6) (1971) 1609-1623.

[17] W. D. Iwan, I.-M. Yang, Application of statistical linearization techniques to nonlinear multi-degree-of-freedom systems, Journal of Applied Mechanics 39 (1972) 545-550.

[18] R. Stratonovich, Topics in the theory of random noise, New york, 1963.

[19] N. Kryloff, N. N. Bogoliubov, Introduction to Non-linear Mechanics, Princeton University Press, 1947.

[20] J. Roberts, P. Spanos, Stochastic averaging: An approximate method of solving random vibration problems, International Journal of Non-Linear Mechanics 21 (2) (1986) 111 - 134.

[21] W. Q. Zhu, Stochastic averaging methods in random vibration, Applied Mechanics Reviews 41 (5) (1988) 189.

[22] I. Elishakoff, P. Colajanni, Stochastic linearization critically re-examined, Chaos, Solitons and Fractals 8 (12) (1997) 1957-1972.

[23] P. Colajanni, I. Elishakoff, A subtle error in conventional stochastic linearization techniques, Chaos, Solitons and Fractals 9 (3) (1998) 479-491. doi:10.1016/S0960-0779(97)00086-6.

[24] P. Bernard, L. Wu, Stochastic linearization: The theory, Journal of Applied Probability 35 (3) (1998) 718-730.

[25] P. Bernard, Stochastic linearization: What is available and what is not, Computers and Structures 67 (1-3) (1998) 9-18.

[26] L. Socha, T. Soong, Linearization in analysis of nonlinear stochastic systems, Applied Mechanics Reviews 44 (10) (1991) $399-422$.

[27] L. Socha, Linearization in analysis of nonlinear stochastic systems: Recent results - part i: Theory, Applied Mechanics Reviews 58 (1-6) (2005) 178-205.

[28] L. Socha, Linearization in analysis of nonlinear stochastic systems, recent results - part ii: Applications, Applied Mechanics Reviews 58 (1-6) (2005) 303-314.

[29] M. R. Torres, C. D. Mote, Expected equivalent damping under random excitation, Journal of Engineering for Industry 91 (4) (1969) 967-973.

[30] L. Chuangdi, L. Tun, B. Dingwei, G. Xinguang, Equivalent damping of sdof structure with maxwell damper, Earthquake Engineering and Engineering Vibration 17 (3) (2018) 627-639.

[31] K. Worden, G. R. Tomlinson, Nonlinearity in Structural Dynamics: Detection, Identification and Modelling, CRC Press, 2000.

[32] P. Eykhoff, System Identification, Parameter and State Estimation, Wiley, New York, 1974. 
[33] M. Enqvist, L. Ljung, Linear approximations of nonlinear fir systems for separable input processes, Automatica 41 (3) (2005) 459-473.

[34] R. Pintelon, J. Schoukens, System identification: A frequency domain approach, second edition, System Identification: a Frequency Domain Approach, Second Edition.

[35] J.-W. Liang, B. F. Feeny, Balancing energy to estimate damping parameters in forced oscillators, Journal of Sound and Vibration 295 (3) (2006) $988-998$.

[36] J.-W. Liang, Damping estimation via energy-dissipation method, Journal of Sound Vibration 307 (2007) 349 - 364.

[37] B. F. Feeny, Estimating damping parameters in multi-degree-of-freedom vibration systems by balancing energy, Journal of Vibration and Acoustics, Transactions of the ASME 131 (4) (2009) $410051-410057$.

[38] R. Brincker, C. Ventura, Introduction to Operational Modal Analysis, Wiley, 2015.

[39] R. W. Clough, J. Penzien, Dynamics of Structures, Computers \& Structures, Inc. - Berkeley, 1995.

[40] D. Newland, An introduction to random vibrations, spectral and wavelet analysis, Longman Scientific \& Technical, 1993.

[41] A. Naess, T. Moan, Stochastic Dynamics of Marine Structures, Cambridge University Press, 2012.

[42] H. M. James, N. B. Nichols, R. S. Phillips, Theory of servomechanisms, Massachusetts Institute of Technology Radiation Laboratory Series 25 (1947) n. pag.

[43] K. S. Mohammad, K. Worden, G. Tomlinson, Direct parameter-estimation for linear and non-linear structures, Journal of Sound and Vibration 152 (3) (1992) 471-499.

[44] C. Lawson, R. Hanson, Solving least squares problems, Prentice-Hall, 1974

[45] J. O'Callahan, P. Avitabile, R. Riemer, System equivalent reduction and expansion process, Proceedings of the 7th International Modal analysis conference, Society of Experimental Mechanics (1989) 29-37.

[46] W. D. Iwan, A distributed-element model for hysteresis and its steady-state dynamic response, Journal of Applied Mechanics 33 (4) (1966) 893.

[47] L.-Y. Lu, L.-L. Chung, L.-Y. Wu, G.-L. Lin, Dynamic analysis of structures with friction devices using discrete-time state-space formulation, Computers \& Structures 84 (1516) (2006) 1049 - 1071.

[48] R. J. Allemang, The modal assurance criterion - twenty years of use and abuse, Journal of Sound and Vibration 37 (8) (2003) 14-21. 